\title{
Conductivity and diffusion near the percolation threshold
}

\author{
A Coniglio $†+, M$ Daoud $\dagger$ and $\mathrm{H} J$ Herrmann $\$ \|$ \\ $\uparrow$ Laboratoire Léon Brillouinf, Centre d’Etudes Nucléaires de Saclay, 91191 Gif-sur-Yvette \\ Cedex, France \\ $\S$ HLRZ, KFA Julich, Postfach 1913, 5170 Julich, Federal Republic of Germany
}

Received 22 March 1989

\begin{abstract}
We consider the scaling properties of the conductivity for non-zero frequencies. We analyse the relevance of various models to descriptions of actual experimental situations. We discuss more particularly the equivalence between conduction and diffusion. In this case, we find a regime where the conductivity is proportional to the size $L$ of the sample, corresponding to an anomalous skin effect. This $L$ dependence leads to a frequencydependent conductivity different from the result of Gefen, Aharony and Alexander. We finally introduce a model consisting of a two-dimensional random resistor system lying on a three-dimensional substrate made of capacitors. This might be relevant to describe a system of conducting particles deposited on a thin insulating substrate such as considered recently by Laibowitz and Gefen.
\end{abstract}

\section{Introduction}

Time-dependent conductivity near the percolation threshold $p_{\mathrm{c}}$ has attracted considerable attention this past decade. This is related to the many potential applications in a variety of phenomena ranging from conduction in random porous media such as oil reservoirs to viscoelastic properties of polymers and gels. In spite of the simplicity of the percolation model, however, different approaches were used for these dynamical properties. These may be roughly divided into two categories. Historically, Efrös and Schkloskii (ES) provided the first theoretical approach [1], which was subsequently used and developed by many others [2-6]. The important result here is that there is a characteristic frequency $\Omega \sim\left|p-p_{\mathrm{c}}\right|^{s+t}$ that vanishes with an exponent related to both exponents $s$ and $t$ of the random superconductor and the random resistor network respectively. For high frequencies, $\omega \gg \Omega$, both the real and imaginary parts of the conductivity diverge as $\operatorname{Re} \Sigma \sim \operatorname{Im} \Sigma \sim \omega^{u}$ with $u=t /(s+t)$. For low frequencies $(\omega \ll$ $\Omega)$ the exponent $s$ also describes the divergence of the dielectric constant $\varepsilon_{\mathrm{r}}=$ $(\operatorname{Im} \Sigma) / \omega \sim\left|p-p_{\mathrm{c}}\right|^{-s}$ as $p$ approaches $p_{\mathrm{c}}$ in the insulating regime.

More recently, Gefen, Aharony and Alexander (GAA) [7] provided a quite different approach. This is based on the idea that conduction along a fractal is related to diffusion via the Einstein relation. Then depending on the problem that one considers, only one of the exponents $s$ or $t$ appears in the characteristic frequency $\Omega$. These correspond respectively to the so-called termite [8] or ant [9] diffusion problems. In

\footnotetext{
$¥$ Permanent address: Dipartimento di Fisica, Mostra d’Oltremare, Pad 19, I-80125 Napoli, Italy.

|| Permanent address: Service de Physique Thérorique, Centre d'Etude Nucléaires de Saclay, F-91191 Guf-sur-Yvette Cedex, France.

I Laboratoire commun CNRS-CEA.
} 
the ant approach, for instance, one finds $\Omega \sim\left|p-p_{\mathrm{c}}\right|^{s^{\prime}+t}$ with $s^{\prime}=2 \nu-\beta$, where $\nu$ and $\beta$ are the connectedness length and order parameter exponents in the percolation problem. The large frequency behaviour of the conductivity is characterised by an exponent $u=t /\left(t+s^{\prime}\right)$. In the low-frequency regime the dielectric constant diverges with the exponent $s^{\prime}$ which coincides with the random superconducting exponent $s$ only in mean field.

On the experimental side the situation is not so clear either. Kubovy and Stephan [10] measured the high-frequency conductivity in a three-dimensional mixture of insulating and conducting materials at the percolation threshold and found a value for the exponent $u \simeq 0.72$, in good agreement with the Es prediction $u=t /(s+t) \simeq 0.72$ obtained using the recent estimates for $s$ and $t$ in three dimensions $[11,12]]$. For other three-dimensional experiments see the recent review by Niklasson [13].

On the other hand, the two-dimensional experimental results of Laibowitz and Gefen [14] on thin Au films deposited on an insulating substrate give $u=0.95$, which does not agree either with the ES prediction $u=0.5$ or with the GAA prediction $u=0.35$.

The aim of this paper is to find the conditions of validity of the ES and GAA approaches. To do so, we will express both of them in terms of resistor and capacitor models in order to show more clearly how they relate to actual experimental realisations.

In the following, we will first recall the ES approach, which applies to a random mixture of poor and good conductors and is simply modelled by having a mixture of resistors with probability $p$ and capacitors with probability $(1-p)$. Then we will analyse the GAA analogy between diffusion and conduction. This is equivalent to considering a random resistor network, in which a resistor is present with probability $p$ (and absent with probability $(1-p)$ ). But all the sites are now grounded through a constant capacitor $C_{0}$. In the high-frequency regime the main contribution to the conductance comes from the capacitors. Since there are $L^{d}$ capacitors in parallel, where $L$ is the system size and $d$ the Euclidean dimension, the conductance is proportional to $L^{d}$ and the conductivity to $L$. Such anomalous size dependence, that seems to have been overlooked so far, leads to new exponents. In particular we find for the high-frequency behaviour an exponent $u=(t+\nu) /(t+2 \nu-\beta)$, which for $d=2$ gives a value $u \simeq 0.69$, different from the result of Gefen et al [7]. This value is in agreement with the experimental and model results of Rigord and Hulin [15], but is different from the experimental results of [14].

A possible generalisation of the latter model, which might be especially relevant for interpreting experiments such as the Laibowitz and Gefen [14] random deposition of thin gold films near the percolation threshold, is to assume that the resistor percolation problem lies at the interface of a three-dimensional insulating substrate on which the random mixture is deposed. We will assume that the substrate is made of a regular array of capacitors. An important consequence of the presence of this substrate is the appearance of a new set of exponents that has not been considered so far. In particular, we expect for this model a value of $u$ close to 1 , with a possible cross-over to the ES value $u=0.5$ depending on the width of the substrate. Using the transfer matrix approach we have calculated $u$ in the limit of infinite width and found $u=0.85$.

\section{The Efrös-Shklovskii theory}

Let us consider a random bond percolation problem with a mixture of poor conductors, with conductance $\sigma_{1}$, present with probability $p$, and good conductors, with conduct- 
ance $\sigma_{2}$, present with probability $(1-p)$, with $\sigma_{1} \ll \sigma_{2}$. Let $\Sigma\left(\sigma_{1}, \sigma_{2}, \varepsilon\right)$ be the conductance of the mixture at a distance $\varepsilon=p-p_{c}$ from the threshold. Following the notation of Hong et al [4], this may be written in the following scaled form:

$$
\Sigma\left(\sigma_{1}, \sigma_{2}, \varepsilon\right)=\sigma_{1} \varepsilon^{\prime} f_{ \pm}\left(h \varepsilon^{-\phi}\right)
$$

where $h$ is the ratio $\sigma_{2} / \sigma_{1}, f_{ \pm}(x)$ is a scaling function valid above ( + ) or below ( - ) the threshold, and the cross-over exponent $\phi$ was calculated first by Efrös and Shklovskii [1], with the result

$$
\phi=t+s
$$

where $t$ and $s$ are respectively the exponents of the conductivity in the random resistor problem and the random superconductor problem. If we now consider the case when $\sigma_{1}=R^{-1}$ is a resistor and $\sigma_{2}=\mathrm{j} C \omega$ is a capacitor, with $\mathrm{j}=\sqrt{-1}$, relation (1) becomes [16]

$$
\Sigma R=\varepsilon^{t} f_{ \pm}\left(\mathrm{j}\left(\omega / \omega_{0}\right) \varepsilon^{-\phi}\right)
$$

where $\omega_{0}=(R C)^{-1}$ is a microscopic characteristic frequency. Relation (3) exhibits a characteristic frequency $\Omega$

$$
\Omega=\omega_{0} \varepsilon^{\phi}
$$

that vanishes when one approaches the percolation threshold with the characteristic exponent $\phi$.

For $\omega \gg \Omega$, the $\varepsilon$ dependence disappears and $\Sigma R \sim \omega^{u} \exp (\mathrm{j} u \pi / 2)$ with $u=$ $t /(s+t)$. We now turn to the GAA approach which is based on the Einstein relation between conductivity and diffusion coefficient [17].

\section{The diffusion equivalence}

This approach does not explicitly take into account the presence of the capacitors as in the Es theory, and therefore is difficult to relate to experimental realisations. Here we wish to find the network model, made of resistors and capacitors, which is equivalent to the GAA approach and calculate its frequency-dependent conductivity. Surprisingly, the result differs from that of Gefen et al. This discrepancy is due to the fact that in the GAA approach it was not taken into account that the time-dependent conductivity scales linearly with the system size $L$. While this size dependence may be seen easily in the resistor-capacitor network model, it is somewhat hidden in the diffusion approach. We start with the classical ant problem, i.e. a particle diffusing on a network. The master equation for such a random walk is

$$
\sum_{j}\left(P_{i}-P_{j}\right) W_{i j}=\stackrel{\circ}{P}_{i}
$$

where $P_{i}$ is the density of walkers at site $i, \dot{P}_{i}$ its derivative with respect to time, $W_{i j}$ is the transition probability from site $i$ to site $j$, and the sum is over all nearest neighbours $j$ to site $i$.

Consider now a resistor network made of a conductance $\sigma_{i}$ between nearestneighbour sites and where every site is grounded through a capacitor $C_{0}$ (see figure 1). The equation for the voltage at each node is given by

$$
\sum_{j}\left(V_{i}-V_{j}\right) \sigma_{i j}=C_{0} \dot{\circ}_{i}
$$




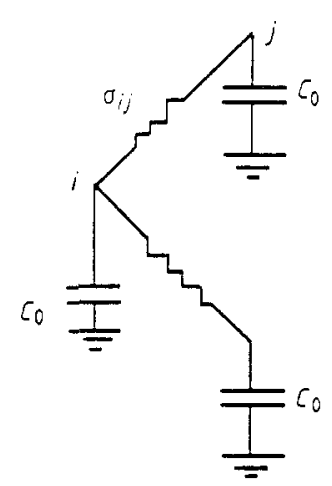

Figure 1. The equivalence between conduction and diffusion is based on a random resistor network where the sites are grounded through a capacitor.

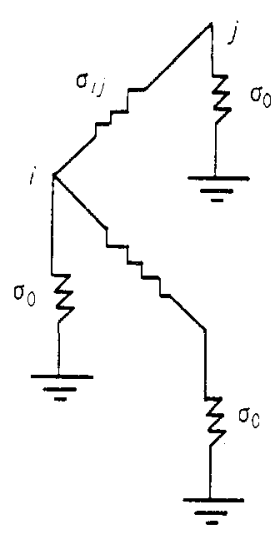

Figure 2. Modification of figure 1 where the capacitors are replaced by conductances.

which is identical to equation (5) for the random walkers. Because of this correspondence, the time-dependent diffusion coefficient in the random walk problem is related via the Einstein relation to the conductivity of the random network made of resistors grounded through the capacitors. Let us then calculate the conductivity for the system shown in figure 1 and then compare it with the diffusion coefficient for the random walk problem. Note that equation (6) can also be written as

$$
\sum\left(\tilde{V}_{j}-\tilde{V}_{i}\right) \sigma_{i j}=\mathrm{j} \omega C_{0} \tilde{V}_{i}
$$

where $\tilde{V}_{i}$ is the Fourier transform of the voltage with respect to time. In the following, we wish to study the scaling behaviour of the conductance of the system in figure 1 as a function of its size $L$. To do so, we first consider the system shown in figure 2 . This is obtained from figure 1 by replacing the capacitors with pure conductances $\sigma_{0}$. The equations for the voltage are given by

$$
\sum\left(V_{j}-V_{i}\right) \sigma_{i j}=\sigma_{0} V_{i}
$$

Comparing relations (7) and (8), we can see that if we find the conductance $G\left(\sigma, \sigma_{0}\right)$ for the latter system, we get the conductance of the system of figure 1 by analytical continuation of $\sigma_{0}$ to the pure imaginary value $\mathrm{j} \omega C_{0}$.

We first consider the case in which all the conductances are present and identical, $\sigma_{i j}=\sigma$, and calculate the conductance $\sigma_{n}$ for a one-dimensional system of length $n$. It is easy to derive the following recursion for $n>1$ :

$$
\sigma_{n}=\sigma_{0}+\sigma \sigma_{n-1} /\left(\sigma+\sigma_{n-1}\right)
$$

with

$$
\sigma_{1}=\sigma+\sigma_{0}
$$

For $n \rightarrow \infty$, the above equation leads to the following solution:

$$
2 \sigma_{\infty}=\sigma_{0}+\sqrt{\sigma_{0}^{2}+4 \sigma \sigma_{0}}
$$

which is finite. Therefore, for a large value of the system size $L$, the conductivity $\sigma_{L} \simeq \sigma_{\infty}$ is independent of $L$ except when $\sigma_{0}=0$ or $\sigma=\infty$. For $\sigma_{0}=0$ we have $\sigma_{L}=L^{-1} \sigma$, 
whereas for $\sigma=\infty$ we have $\sigma_{L}=\infty$. However, if one of the bonds $\sigma$ is kept finite, which is the case that we will consider, then $\sigma_{L}=L \sigma_{0}$.

Thus in general we expect the following scaling form for a one-dimensional system of size $L$ :

$$
\sigma_{L}=L \sigma_{0} f\left(h L^{2}\right)
$$

where $h=\sigma_{0} / \sigma$, and

$$
f(x) \sim \begin{cases}f_{0}+f_{1} / x & x \ll 1 \\ x^{-1 / 2} & x \gg 1\end{cases}
$$

where $f_{0}$ and $f_{1}$ are constants.

For a $d$-dimensional system, the conductance $G$ is $G=L^{d-1} \sigma_{L}$, since it is made of $L^{d-1}$ one-dimensional channels of conductance $\sigma_{L}$ in parallel. Thus the conductivity $\Sigma$ for a $d$-dimensional network in which all sites are grounded (see figure 2 ) is

$$
\Sigma=G / L^{d-2}=L^{2} \sigma_{0} f\left(h L^{2}\right)
$$

where $f(x)$ is given by (11).

Analytically continuing to imaginary values $\sigma_{0}=\mathrm{j} \omega C_{0}$, we get

$$
\Sigma=L^{2} \mathrm{j} \omega C_{0} f\left(\mathrm{j} \omega C_{0} L^{2} / \sigma\right)
$$

which, for large frequencies, $\omega \rightarrow \infty$, gives, from $(11 b)$,

$$
\Sigma=L \sigma^{1 / 2}\left(j \omega C_{0}\right)^{1 / 2}
$$

Let us now consider the dilute case of the system in figure 2 in $d$ dimensions, in which $\sigma_{i j}=\sigma$ with probability $p$ and zero with probability $(1-p)$. At the percolation threshold, we will show the asymptotic behaviours are as follows:

$$
\Sigma\left(\sigma, \sigma_{0}\right) \sim \begin{cases}\sigma L^{-\tilde{t}} & \sigma_{0}=0 \\ \sigma_{0} L^{2-\tilde{\beta}} & \sigma=\infty \\ \sigma_{0}^{u} \sigma^{1-u} L & \sigma_{0} \text { and } \sigma \text { finite }\end{cases}
$$

where $\tilde{t}=t / \nu$ and $\tilde{\beta}=\beta / \nu$ are respectively the conductivity and order parameter exponents divided by the connectedness length exponent, and

$$
u=(1+\tilde{t}) /(2+\tilde{t}-\tilde{\beta}) \text {. }
$$

The behaviour in $(15 b)$ is due to the fact that $L^{D}$ conductances $\sigma$ contribute to the total conductance $G$, with $D$ the fractal dimension. Thus the conductivity behaves as $\Sigma=G / L^{d-2} \sim L^{D-d+2}$. Using the scaling relation $d-D=\tilde{\beta}$, we find $(15 b)$.

The linear dependence on $L$ in (15c) follows from the fact that, for $\sigma$ and $\sigma_{0}$ finite, the $L$ dependence is given by that at $p=1$, equations (11b) and (12).

Relations (15) may be written in the general scaling form

$$
\Sigma\left(\sigma, \sigma_{0}\right)=\sigma_{0} L^{2-\tilde{\beta}} g\left(h L^{\tilde{\phi}}\right)
$$

with

$$
\tilde{\phi}=\tilde{t}+2-\tilde{\beta}
$$


and the asymptotic behaviours

$$
g(x) \sim \begin{cases}g_{0}+g_{1} / x & x \ll 1 \\ x^{u-1} & x \gg 1 .\end{cases}
$$

For $p$ different from $p_{\mathrm{c}}$, the conductivity is given by

$$
\Sigma=L \sigma_{0} \xi^{1-\tilde{\beta}} g_{ \pm}\left(h \xi^{\tilde{\phi}}, \xi / L\right)
$$

where $h=\sigma_{0} / \sigma, \tilde{\phi}$ and $u$ are given in relations (18) and (16) respectively. For $\xi / L \ll 1$, equation (20) gives the following behaviour:

$$
\Sigma \sim \begin{cases}\sigma \xi^{-i} & p \rightarrow p_{c}^{+} ; \sigma_{0}=0 \\ L \sigma_{0} \xi^{1-\tilde{\beta}} & p \rightarrow p_{c}^{-} ; \sigma=\infty \\ L \sigma_{0}^{u} \sigma^{1-u} & h \xi^{\tilde{\phi}} \gg 1 .\end{cases}
$$

The linear dependence in $L$ in relation $(21 b)$ is due to the fact that for $p<p_{\mathrm{c}}$ and $\sigma_{0} \neq 0$ the conductance $G$ is proportional to $(L / \xi)^{d-1} \xi^{D}$ because there are $(L / \xi)^{d-1}$ superconducting clusters in parallel, each connected to the ground through $\xi^{D}$ resistors in parallel. The conductivity is $\Sigma=G / L^{d-2} \sim L \xi^{1-\tilde{\beta}}$. Finally since for $\sigma_{0} \neq 0$ the conductivity is proportional to $L$ above and below $p_{c}$, this same linear dependence follows also at $p_{\mathrm{c}}$, equation $(21 c)$.

Note also that from relation $(15 c)$ we have, for $h L^{2} \gg 1$ and $h \xi^{\tilde{\phi}} \ll 1$, a regime where

$$
\Sigma=L \sigma_{0}^{1 / 2} \sigma^{1 / 2} \quad h L^{2} \gg 1, h \xi^{\tilde{\phi}} \ll 1 .
$$

Analytically continuing $\sigma_{0}$ to $\mathrm{j} \omega C$, we find

$$
\Sigma=L j \omega C \xi^{1-\tilde{\beta}} g_{ \pm}\left(\mathrm{j} \omega C \xi^{\tilde{\phi}} / \sigma, \xi / L\right)
$$

which, for $\xi / L \ll 1$, has the following limits:

$$
\Sigma= \begin{cases}L \mathrm{j} \omega C \xi^{1-\tilde{\beta}} & \omega=0, p \rightarrow p_{\mathrm{c}}^{-} \\ L \sigma^{1 / 2}(\mathrm{j} \omega C)^{1 / 2} & L^{1 / 2} \omega C / \sigma \gg 1 \\ L \sigma^{1-u}(\mathrm{j} \omega C)^{u} & \xi^{\hat{\phi}} \omega C / \sigma \gg 1 .\end{cases}
$$

Note that equation (24) implies a dielectric constant

$$
\varepsilon_{\mathrm{r}}=(\operatorname{Im} \Sigma) / \omega \sim L \xi^{1-\tilde{\beta}} .
$$

These results do not agree with the analysis based on anomalous diffusion. Indeed we find that the dielectric constant diverges with an exponent $s^{\prime}=1-\tilde{\beta}$, equation (27), while the high-frequency behaviour is governed by the exponent $u=(1+\tilde{t}) /(2+\tilde{t}-\tilde{\beta})$, equation (26). We recall that in the anomalous diffusion approach it was found that $s^{\prime}=2-\tilde{\beta}$ and $u=\tilde{t} /(2+\tilde{t}-\tilde{\beta})$. The reason for this discrepancy is that in the ant approach, the linear dependence of the macroscopic conductivity with $L$ for $\omega \neq 0$, relation (27), was not taken into account.

We will show now that, if we use the diffusion approach of Gefen et al and take this size dependence properly into account in the macroscopic conductivity, we reproduce the result $(26)$.

Using the equivalence between diffusion constant and conductivity, Gefen et al found that at $p_{\mathrm{c}}$ the characteristic time $\tau$ for the ant to reach the boundary of a system of linear dimension $L$ is

$$
\tau=L^{2+\tilde{i}-\tilde{\beta}} \text {. }
$$


Since the static conductivity scales as $L^{-i}$ and the frequency-dependent conductivity $\Sigma(L, \omega)$ scales linearly with $L$, we have the following scaling behaviour:

$$
\Sigma(L, \omega) \sim \begin{cases}L^{-i} g(\omega t) \sim L^{-\tilde{t}} & \omega \tau \ll 1 \\ L \omega^{(1+\tilde{t}) /(2+i-\tilde{\beta})} & \omega \tau \gg 1\end{cases}
$$

and we recover equation (26).

Had we required that the conductivity be size independent, we would have obtained the result of Gefen et al.

\section{An alternative model}

In the previous sections we have described the model based on anomalous diffusion in terms of a network of resistors and grounded capacitors. Such a formulation makes it easier to predict the relevance of the model to actual physical systems. One case where this model seems to be relevant is the two-dimensional system of Rigord and Hulin [15]. This is a network of graphite deposited on a thin insulating sheet which is put on a conductor plate. The insulating sheet corresponds to the grounded conductors in the model. The measured frequency dependence of the complex conductance is in good agreement with the predicted laws. They find $\operatorname{Re} \Sigma \sim \operatorname{Im} \Sigma \rightarrow \omega^{u}$, with $u=0.67 \pm 0.02$, in agreement with relation (16), giving $u=0.69$.

We propose now another model which may be relevant in some two-dimensional situations. This is shown in figure 3. It is made of a two-dimensional sheet $L \times L$ of a random conducting-insulating mixture in which a conductance is present with probability $p$ and a capacitor $C_{0}$ with probability $(1-p)$. This plane is put on a three-dimensional network of dimensions $L \times L \times L_{1}$ in which each bond consists of a capacitor $C$. Since the result does not depend crucially on the value of $C_{0}$, we will take $C_{0}=C$ for simplicity. When $L_{1}$ is negligible compared with the two-dimensional connectedness length the system can be considered as two-dimensional and we get the same behaviour as the Efrös-Shklovskii model.

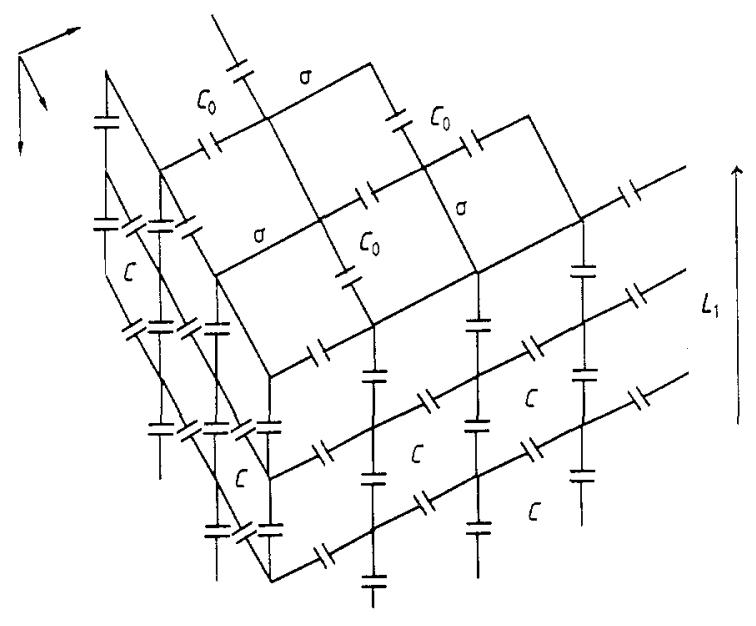

Figure 3. An alternative model consists of a two-dimensional random $\sigma-C_{0}$ system put on a three-dimensional network where all the bonds are capacitors. 
Let us now consider the case when $L_{1} \simeq L$, so that the substrate may be considered as a three-dimensional insulator. As in the previous section, we first consider the case in which the capacitor is replaced by a real conductance $\sigma_{0}$ and then extend the results to complex values of $\sigma_{0}$.

If we fix $p=p_{\mathrm{c}}$, the two-dimensional threshold, the conductance $G$ has the following behaviour:

$$
G \sim \begin{cases}\sigma L^{-\tilde{t}} & \sigma_{0}=0 \\ \sigma_{0} L L^{\tilde{s^{\prime \prime}}} & \sigma=\infty \\ \sigma_{0}^{u} \sigma^{1-u} L & \sigma \text { and } \sigma_{0} \text { finite }\end{cases}
$$

where $\tilde{t}$ is the two-dimensional conductivity exponent which is known to be numerically $\tilde{t}=0.98 . \tilde{s}^{\prime \prime}$ is a new exponent which describes the divergence of a three-dimensional network in which the bonds on the top surface are made either of superconducting links with probability $p$ or finite conductances with probability $(1-p)$. We expect $s^{\prime \prime}$ to be close to zero. This has been confirmed by a calculation that we have done using the transfer matrix approach, which gives $s^{\prime \prime} \simeq 0.15$ (see next section). The extra linear dependence on $L$ is due to the three-dimensional nature of the system whenever $\sigma_{0} \neq 0$. The exponent $u$ will be discussed below.

As in the previous section, relations (30) may be summarised in the following scaling equation:

$$
G=\sigma_{0} L L^{\tilde{s} \prime} g\left(h L^{\tilde{\phi}}\right)
$$

where $h=\sigma_{0} / \sigma$ and the function $g(x)$ has the following asymptotic behaviours:

$$
\begin{array}{ll}
g(x)=D+F / x & x \ll 1 \\
g(x) \sim x^{u-1} & x \gg 1
\end{array}
$$

with $u=(1+\tilde{t}) /\left(\tilde{t}+\tilde{s}^{\prime \prime}\right)$.

Away from $p_{c}$ the conductance is

$$
G=\sigma_{0} L \xi^{\tilde{s}^{\prime \prime}} g_{ \pm}\left(h \xi^{\tilde{\phi}}, \xi L\right)
$$

where $\xi$ is the two-dimensional connectedness length. Analytically continuing $\sigma_{0}$ to $\mathrm{j} \omega C$, we find that the zero-frequency dielectric constant diverges as $\left(p-p_{\mathrm{c}}\right)^{-s^{\prime \prime}}$ while for high frequencies the real and imaginary parts of the conductance diverge as $\omega^{u}$ with

$$
u=(1+\tilde{t}) /\left(1+\tilde{t}+\tilde{s}^{\prime \prime}\right) \simeq 1
$$

because of our estimate for $s^{\prime \prime}$. This last result is consistent with the experimental result of Laibowitz and Gefen [14] on thin Au films deposited on an insulating substrate.

Finally, we note that a three-dimensional mixture of insulating and conducting materials should be well described by the Efrös-Shklovskii model, because of the absence of any substrate. The experiments of Kubovy and Stephan on such a system at $p_{\mathrm{c}}$ are in good agreement indeed with this model.

\section{The exponent $\tilde{s}^{\prime \prime}$}

We calculate the exponent $\tilde{s}^{\prime \prime}$ using the transfer matrix approach $[12,18]$. In this method one constructs an $L \times L \times n$ bar of a cubic lattice by adding bond after bond. In the first vertical plane a voltage $V=0$ is imposed and one looks for the voltages $V_{i}$ 
which should be put on each of the $N^{2}$ end points in the $n$th vertical plane in order that they carry a current $I_{i}$. They are related through the resistance matrix $R_{i j}$ by

$$
V_{i}=\sum_{j=1}^{N} R_{i j} I_{j} \quad i=1,2, \ldots, N
$$

The matrix $R_{i j}$ must be updated for each bond that is added via

$$
R_{i j}^{\prime}=R_{i j}+r \delta_{i \alpha} \delta_{j \alpha}
$$

if the bond is longitudinal at line $\alpha$ and via

$$
R_{i j}^{\prime}=R_{i j}-\frac{\left(R_{i \alpha}-R_{i \beta}\right)\left(R_{\sigma j}-R_{\beta j}\right)}{r+\left(R_{\alpha \alpha}+R_{\beta \beta}-R_{\alpha \beta}-R_{\beta \alpha}\right)}
$$

if the bond is transverse between lines $\alpha$ and $\beta ; r$ is the resistance of the bond. The resistance of the bar in the limit of very long bars $(n \rightarrow \infty)$ is then given by $R=R_{i i} / L$, independent of $i$.

To calculate $\tilde{s}^{\prime \prime}$ we consider a bar which on the upper plane has conductors $(r=1)$ with probability $1-p_{\mathrm{c}}$ and superconductors $(r=0)$ with probability $p_{\mathrm{c}}$, where $p_{\mathrm{c}}=\frac{1}{2}$. All the other bonds are conductors. In the transverse direction, parallel to the plane, we impose periodic boundary conditions. In the transverse direction perpendicular to the plane, we consider two cases:

(A) free boundary conditions, which correspond to a free surface plane on the surface of the bulk;

(B) periodic boundary conditions, which correspond to the case of a surface of defects within the bulk.

Case (A) corresponds to the physical situation described in this paper and should yield $\tilde{s}^{\prime \prime}$. Case (B) is in principle another problem and its exponent $\tilde{s}^{\prime}$ gives an upper bound for the exponent $\tilde{s}^{\prime \prime}$; the advantage of $(B)$ is that due to the periodic boundary conditions, the convergence in $L$ is faster and thus the numerical simulation is more reliable.

In figure 4 we show the result of our calculation. The upper curve corresponds to case (A) and the lower one to case (B). We plot $R$ as a function of the linear size $L$

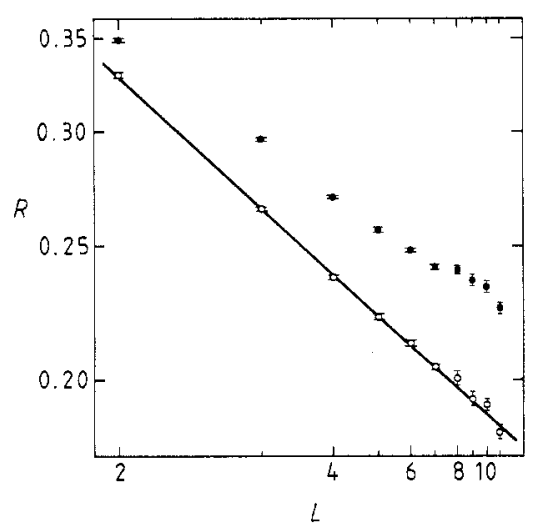

Figure 4. Log-log plot of the resistance $R$ against the width $L$ of the strip. The full circles correspond to open boundary conditions; the open circles correspond to closed boundary conditions.

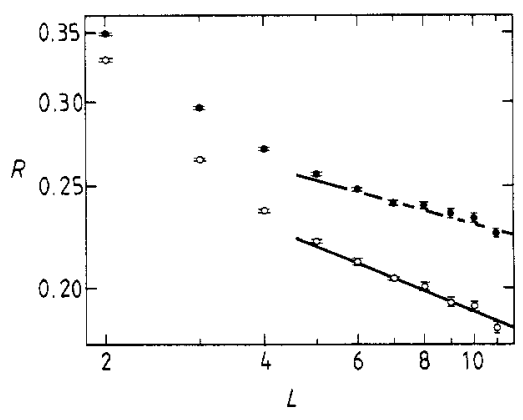

Figure 5. $\ln R$ plotted against $\ln (\ln L)$; symbols have the same meaning as in figure 4 . 
of the bar in a log-log plot, so that the data should, for large enough $L$, fall on straight lines of slopes $-\tilde{s}^{\prime \prime}$ and $-\tilde{s}^{\prime}$ respectively. We have considered $n$ between $10^{5}$ and $10^{6}$ and spent 30 hours on the Cray XMP to obtain these data. The error bars are calculated from the statistical fluctuations between several pieces of the bar. From the straight lines of figure 4 we conclude

$$
\tilde{s}^{\prime \prime}=0.15 \pm 0.02 \quad \tilde{s^{\prime}}=0.21 \pm 0.02 .
$$

We investigated the same data also for the possibility of a logarithmic decay of the form

$$
R \sim(\ln L)^{\tilde{x}} .
$$

From figure 5 we see that the data are also not incompatible with such a behaviour with

$$
\tilde{x}^{\prime \prime}=0.3 \pm 0.1 \quad \tilde{x}^{\prime}=0.42 \pm 0.04
$$

\section{Conclusion}

We have considered three models to describe the frequency dependence of the conductivity at the percolation threshold.

The first one, due to Efrös and Shklovskii [1], is made of resistors present with probability $p$ and capacitors with probability $(1-p)$ and is expected to apply to three-dimensional systems, as found in [16]. For high frequencies in particular, it gives $\Sigma \sim \omega^{u}$, with $u=\tilde{t} /(\tilde{t}+\tilde{s})$.

The second model is based on the anomalous diffusion model of Gefen et al and is made of finite resistors present with probability $p$ and infinite resistors present with probability $(1-p)$. All the resistors are now grounded via a capacitor. A typical experimental realisation is the system of Rigord and Hulin [15] which consists of a two-dimensional random resistor network on top of a two-dimensional insulating sheet which lies on a conducting substrate. For high frequencies it predicts $u=$ $(1+\tilde{t}) /(\tilde{t}+2-\tilde{\beta})$, in agreement with the experimental data. This result differs from that given in the original paper [7], based on the anomalous diffusion. The discrepancy is due to a factor $L$ in the frequency-dependent conductivity which was overlooked in the original approach.

Finally we introduced a third model here, which also applies to the two-dimensional random conductor-insulator composite materials, when the substrate is three dimensional. This model predicts a value for $u$ slightly smaller than unity, as found in the experiment of Laibowitz and Gefen.

All three models are defined in terms of resistors and capacitors and it should be easy to decide which one should apply in any experimental realisation. For instance we expect that by changing the conducting substrate into an insulating one in the Rigord-Hulin experiment, one should observe the result given by model 3 , name $u=1$.

\section{Acknowledgments}

The authors are much indebted to L De Arcangelis, R Bidaux and J M Luck for related discussions. 


\section{References}

[1] Efrös A L and Shklovskii B I 1976 Phys. Status Solidi b 76131

[2] Straley J 1977 Phys. Rev. B 155733

[3] Webman I, Jortner J and Cohen M H 1977 Phys. Rev. B 162593

[4] Hong D C, Stanley H E, Coniglio A and Bunde A 1986 Phys, Rev. B 334564

[5] Clerc J P, Giraud G, Laugier J M and Luck J M 1985 Physics of Finely Divided Matter (Proceedings in Physics 5) (Berlin: Springer) p 193

[6] Bug A L R, Grest G S, Cohen M H and Webman I 1987 Phys. Rev. B 363675

[7] Gefen Y, Aharony A and Alexander S 1983 Phys. Rev. Lett. 5077

[8] Coniglio A and Stanley H E 1984 Phys. Rev. Lett. 521068

[9] Havlin S and Ben Avraham D 1987 Adv. Phys. 36695

[10] Kubovy A and Stephan O 1986 Thin Solid Films 135 L9

[11] Derrida B, Stauffer D, Herrmann H J and Vannimenus J 1983 J. Physique Lett. 44701

[12] Herrmann H J, Derrida B and Vannimenus J 1984 Phys. Rev. B 304080

[13] Niklasson G A 1987 J. Appl. Phys. 621

[14] Laibowitz R G and Gefen Y 1984 Phys, Rev. Lett. 53380

[15] Rigord P and Hulin J P 1988 Europhys. Lett. 6145

[16] Daoud M, Hong D and Family F 1988 J. Phys. A: Math. Gen. 21 L917

[17] de Gennes P G 1980 J. Physique 43 C3-17

[18] Derrida B and Vannimenus J 1982 J. Phys A: Math. Gen. 15 L557 